\title{
Loss of PIP5Kl $\gamma$, unlike other PIP5KI isoforms, impairs the integrity of the membrane cytoskeleton in murine megakaryocytes
}

Yanfeng Wang, ${ }^{1}$ Rustem I. Litvinov, ${ }^{2}$ Xinsheng Chen, ${ }^{1}$ Tami L. Bach, ${ }^{1}$ Lurong Lian, ${ }^{1}$ Brian G. Petrich, ${ }^{3}$ Susan J. Monkley, ${ }^{4}$ David R. Critchley, ${ }^{4}$ Takehiko Sasaki, ${ }^{5}$ Morris J. Birnbaum, ${ }^{1}$ John W. Weisel, ${ }^{2}$ John Hartwig, ${ }^{6}$ and Charles S. Abrams ${ }^{1}$

\begin{abstract}
${ }^{1}$ Department of Medicine and ${ }^{2}$ Department of Cell and Developmental Biology, University of Pennsylvania, Philadelphia, Pennsylvania, USA. ${ }^{3}$ Department of Medicine, UCSD School of Medicine, La Jolla, California, USA. ${ }^{4}$ Department of Biochemistry, University of Leicester, Leicester, United Kingdom. ${ }^{5}$ Department of Pathology and Immunology, Akita School of Medicine, Akita, Japan. ${ }^{6}$ Hematology Division, Brigham and Women's Hospital, Boston, Massachusetts, USA.
\end{abstract}

\begin{abstract}
Phosphatidylinositol-4,5-bisphosphate (PIP $)$ is an abundant phospholipid that contributes to second messenger formation and has also been shown to contribute to the regulation of cytoskeletal dynamics in all eukaryotic cells. Although the $\alpha, \beta$, and $\gamma$ isoforms of phosphatidylinositol-4-phosphate-5-kinase I (PIP5KI) all synthesize PIP2, mammalian cells usually contain more than one PIP5KI isoform. This raises the question of whether different isoforms of PIP5KI fulfill different functions. Given the speculated role of PIP ${ }_{2}$ in platelet and megakaryocyte actin dynamics, we analyzed murine megakaryocytes lacking individual PIP5KI isoforms. PIP5 KI $\gamma^{-/-}$megakaryocytes exhibited plasma membrane blebbing accompanied by a decreased association of the membrane with the cytoskeleton. This membrane defect was rescued by adding back wildtype PIP5KI $\gamma$, but not by adding a catalytically inactive mutant or a splice variant lacking the talin-binding motif. Notably, both PIP5KI $\beta$ - and PIP5KI $\gamma^{-/-}$cells had impaired PIP 2 synthesis. However, PIP5KI $\beta$-null cells lacked the membrane-cytoskeleton defect. Furthermore, overexpressing PIP5KI $\beta$ in PIP5KI $\gamma^{-/-}$cells failed to revert this defect. Megakaryocytes lacking the PIP5KI $\gamma$-binding partner, talin1, mimicked the membrane-cytoskeleton defect phenotype seen in PIP5 $\mathrm{KI}^{-/-}$cells. These findings demonstrate a unique role for PIP5KI $\gamma$ in the anchoring of the cell membrane to the cytoskeleton in megakaryocytes, probably through a pathway involving talin. These observations further demonstrate that individual PIP5KI isoforms fulfill distinct functions within cells.
\end{abstract}

\section{Introduction}

In contrast to the head group of other phospholipids, the head group of phosphatidylinositol can be phosphorylated reversibly at the 3,4 , or 5 position to generate a family of phosphoinositides. This process was initially described by Mabel and Lowell Hokin over 40 years ago and is essential to signal transduction within all eukaryotic cells (1). Arguably, the best-studied phosphoinositide signaling pathway is the hydrolysis of phosphatidylinositol-4,5bisphosphate $\left(\mathrm{PIP}_{2}\right)$ by phospholipase $\mathrm{C}$ in the synthesis of the second messengers inositol-1,4,5-trisphosphate $\left(\mathrm{IP}_{3}\right)$ and diacylglycerol (DAG) (2-4). For many years, this was assumed to be the sole function of this phosphoinositide. However, $\mathrm{PIP}_{2}$ was subsequently identified as a substrate for phosphatidylinositol-3'-kinase in the generation of additional second messengers. More recently, it has been demonstrated that $\mathrm{PIP}_{2}$ directly binds and regulates the activity of cytoskeletal proteins such as profilin and gelsolin (5-10). By directly binding proteins, $\mathrm{PIP}_{2}$ regulates signaling cascades required for diverse cellular processes, such as actin assembly and vesicle trafficking.

The canonical pathway that leads to $\mathrm{PIP}_{2}$ synthesis is mediated by the phosphorylation of phosphatidylinositol-4-phosphate by

Nonstandard abbreviations used: $\mathrm{PIP}_{2}$, phosphatidylinositol-4,5-bisphosphate; PIP5KI, phosphatidylinositol-4-phosphate-5-kinase I.

Conflict of interest: The authors have declared that no conflict of interest exists. Citation for this article: J. Clin. Invest. 118:812-819 (2008). doi:10.1172/JCI34239. phosphatidylinositol-4-phosphate-5-kinase I (PIP5KI) (11-13). Three genes encode the 3 isoforms of PIP5KI, known as PIP5KI $\alpha$, PIP5KI $\beta$, and PIP5KI $\gamma$. In addition, PIP5KI $\gamma$ has 2 splice variants. Although they are all capable of synthesizing $\mathrm{PIP}_{2}$, these isoenzymes have significantly dissimilar primary structures, are expressed at different levels in different tissues, and appear to localize within different compartments within the cell (14-20). For example, PIP5KI $\alpha$ localizes in membrane ruffles (15), while PIP5KI $\beta$ localizes near endosomes (16), and PIP5KI $\gamma$ is targeted to focal adhesions and nerve terminals (17-19). Since most cells possess more than 1 isoform of PIP5KI, it appears likely that these isoforms have overlapping, but not identical, functions.

In most cells, it is thought that the total concentration of $\mathrm{PIP}_{2}$ neither rises nor falls significantly after agonist stimulation (21). Since the same agonists stimulate the consumption of $\mathrm{PIP}_{2}$ by phospholipase $\mathrm{C}$ and phosphatidylinositol-3'-kinase, this implies that rapid (and perhaps localized) synthesis of $\mathrm{PIP}_{2}$ must occur to compensate for these losses. Recently, some evidence has suggested that local synthesis of $\mathrm{PIP}_{2}$ occurs within discrete regions of the cell membrane $(17,18,22,23)$. However, definitive evidence of localized $\mathrm{PIP}_{2}$ production has been difficult to obtain. We speculate that the different isoforms of PIP5KI are activated within different subdomains of cells. This allows individual isoforms to generate $\mathrm{PIP}_{2}$ within discrete regions of the cell that fulfills specific functions. To test this hypothesis, we studied cells lacking individual isoforms of PIP5KI. 
A

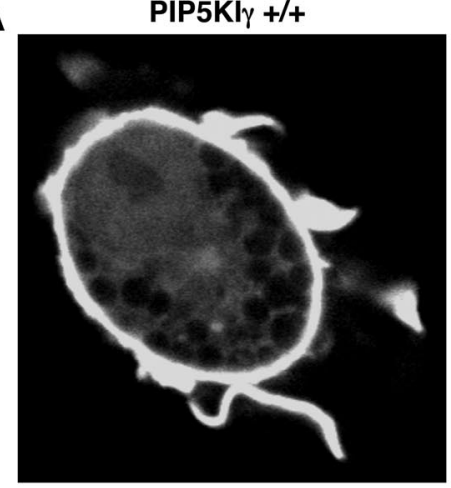

B

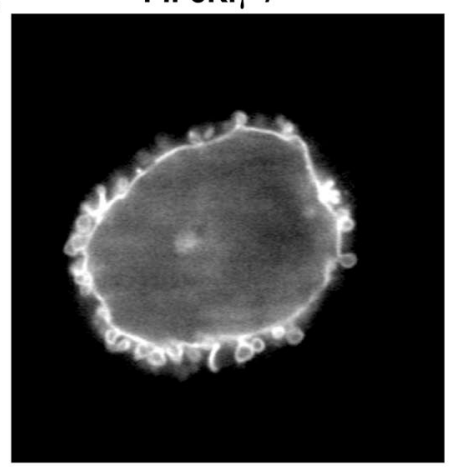

Figure 1

PIP5Kl $\gamma^{-1-}$ megakaryocytes have defective attachment of the cell membrane to the cytoskeleton. Yolk sac progenitor cells were differentiated into megakaryocytes by culturing in thrombopoietin for 5 days. The membranes of wild-type (A) and PIP5KI $\gamma^{-1-}$ (B) megakaryocytes were stained with GFP-

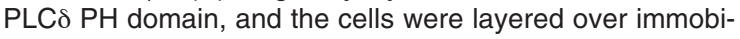
lized fibrinogen. Shown are confocal images of the plasma membrane morphology of PIP5KI $\gamma^{+/+}$and PIP5KI $\gamma^{-/-}$megakaryocytes. Similar results were seen when fluorescent nonspecific lipophilic dye, Vybran DIO, was substituted for GFP$\mathrm{PLC} \delta \mathrm{PH}$. Real-time images of cells can also been seen in Supplemental Movies 1 and 2.

\section{Results}

Platelets, like other hematopoietic cells, undergo rapid bursts of both $\mathrm{PIP}_{2}$ synthesis and actin cytoskeletal reorganization following agonist stimulation (24). We have previously shown that PIP5KI $\beta$ and PIP5KI $\gamma$ are the dominant type I PIP5K isoforms in platelets responsible for $\mathrm{PIP}_{2}$ synthesis (25). Given the hypothesized role for $\mathrm{PIP}_{2}$ synthesis in platelet actin dynamics (24), and the known role of PIP5KI $\gamma$ in cytoskeletal signaling (25), we generated a murine line containing a null mutation within the PIP5KI $\gamma$ gene (26). Although an independently derived PIP5KI $\gamma$-knockout line dies perinatally (27), we found that mice homozygous for the PIP5 $\mathrm{KI} \gamma^{-/-}$mutation died at E11.5. This early prenatal lethality of the PIP5 $\mathrm{KI}^{-/-}$embryos precluded studies of hematopoietic cells derived from the bone marrow or liver (26). Therefore, we analyzed yolk sac progenitor cells that were treated with thrombopoietin ex vivo and differentiated into megakaryocytes. After 5 days in culture, many of the nonadherent cells were multinucleated, and approximately $70 \%$ expressed CD41, a marker for the megakaryocyte lineage. This demonstrated that the majority of yolk sac progenitor cells had differentiated into platelet precursor cells and that PIP5KI $\gamma$ is not required for this differentiation.

Megakaryocytes derived from PIP5KI $\gamma^{-/-}$embryos were layered over immobilized fibrinogen for 30 minutes, and F-actin levels were quantitated after phalloidin staining. There was no evidence of an effect of the PIP5 $\mathrm{KI}^{-/-}$mutation on the quantities of total F-actin in resting and stimulated fibrinogen-adherent megakaryocytes or in cells that were analyzed in suspension in the absence of integrin stimulation (data not shown.)

Impaired membrane-cytoskeletal association in PIP5KI $\gamma^{-1-}$ megakaryocytes. To investigate the effects on actin dynamics, the extension and contraction of membranes during cell adhesion of wild-type and knockout megakaryocytes was observed in real time. Wildtype megakaryocytes (Figure 1A and Supplemental Movie 1; supplemental material available online with this article; doi:10.1172/ JCI34239DS1) and PIP5KI $\gamma^{+/-}$megakaryocytes (data not shown) actively formed and contracted lamellipodia and rapidly spread upon the immobilized matrix. In contrast, PIP5KI $\gamma^{-/-}$megakaryocytes extended membrane blebs rather than normal sheet-like lamellipodia (Figure 1B and Supplemental Movie 2). The cells continuously extended and contracted these membrane blebs but eventually spread as much as wild-type cells. Since membrane blebs were not seen in PIP5KI $\beta^{-/-}$or PIP5 $\mathrm{KI}^{+/-}$megakaryocytes (data not shown), this phenotype was specific to the loss of PIP5KI $\gamma$.

This blebbing phenotype was similar to that seen in a well-characterized M2 melanoma cell line with defective anchoring of the membrane to the cytoskeleton due to a lack of filamin A (28). This observation is also consistent with the previous suggestion that $\mathrm{PIP}_{2}$ contributes to the stable association of the membrane with the cytoskeleton (29). Therefore, we reasoned that PIP5 KI $\gamma^{-/-}$megakaryocytes might have a defect in their ability to anchor their membranes to the cytoskeleton, accounting for the blebbing phenotype. To analyze these cells for a failure to anchor their membranes, we used laser tweezers to mechanically pull the cell membrane apart from the cytoskeleton. A fibrinogen-coated microscopic latex bead moved by a focused laser beam was allowed to repeatedly touch a megakaryocyte attached to a poly-L-lysine-coated glass surface. When the fibrinogen-coated bead interacted and attached to a cell, it either formed an irreversible contact or the bead was capable of being ruptured away from the cell with the forces generated by the laser trap (up to $150 \mathrm{pN}$.) Wild-type megakaryocytes and platelets (data not shown) had rigid membranes that resisted stretching by surface-bound beads that were pulled away from the membrane by the optical trap. The upper tracing in Figure 2A demonstrates the variable forces (downward signals) required to detach the fibrinogen-coated bead from the cell membrane upon repeated touching and separation. Observation of this interaction in real-time demonstrated that at all times the wild-type megakaryocyte membrane remained rigid (Supplemental Movie 3).

Fibrinogen-coated beads also bound PIP5KI $\gamma^{-/-}$megakaryocytes, but these cells did not exhibit rigid membranes. Instead, the membrane of PIP5 $\mathrm{KI}^{-/-}$megakaryocytes had enough flexibility to allow it to remain attached to the bead when it was pulled away from the cell. As a result, the membrane formed an elongating tether that extended from the cell body to the fibrinogen-coated bead (Figure $2 \mathrm{~B})$. In the lower tracing of Figure $2 \mathrm{~A}$, the first downward signal reflected the moment of tether formation by the oscillating bead. Therefore, its magnitude was equivalent to the force needed to tear the membrane part off of the underlying cytoskeletal proteins. The average force required to disrupt the membrane from the cytoskeleton of a PIP5KI $\gamma^{-/-}$megakaryocyte was $63 \pm 16 \mathrm{pN}(n=17)$. This value is commensurate with the forces required to disrupt the membrane-cytoskeleton connection in a neutrophil (30). Since wild-type megakaryocytes and platelets have rigid membranes that resist tethering, a comparable number cannot be determined for these cells within the power limit of the optical trap.

The interaction between fibrinogen-coated beads and PIP5KI $\gamma^{-/-}$ megakaryocytes was further observed in real time and demonstrated that laser beam-induced oscillation of the bead resulted in simultaneous cooscillation of the membrane tether (Supplemental Movie 4). If a PIP5 $\mathrm{KI}^{-/-}$cell tethered to the trapped fibrinogen- 

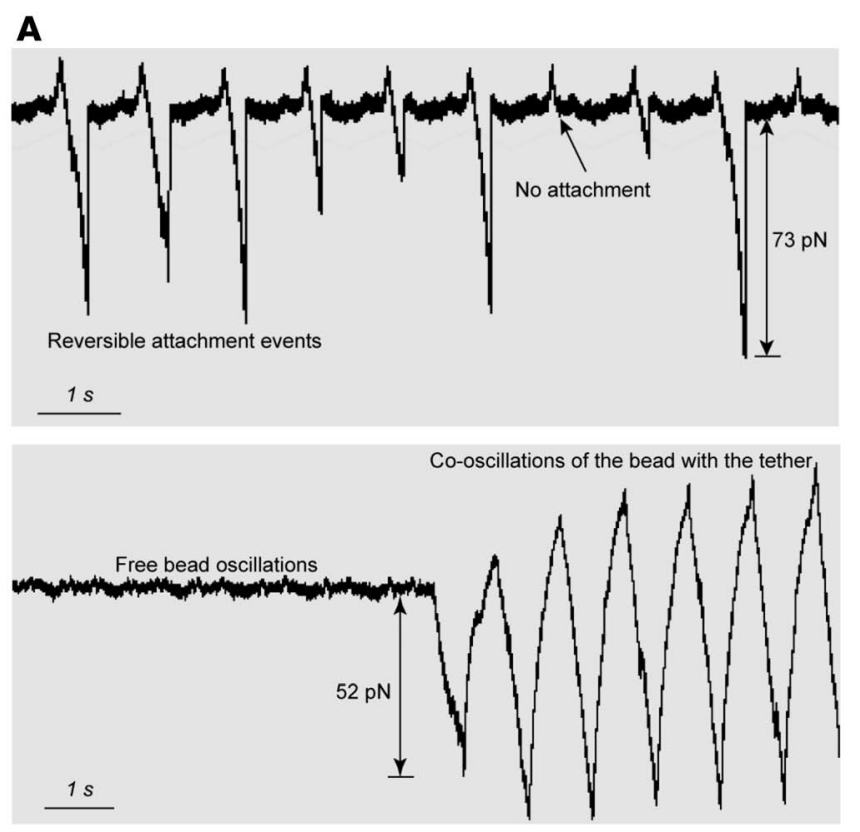

B

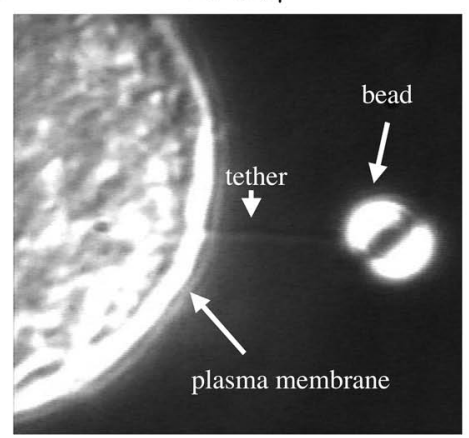

coated bead was moved away from the trapped bead up to several tens of micrometers, the bead ultimately sprang back toward the cell once the laser trap was released (Supplemental Movie 5). The percentages of tether formation in analyzed cells are shown in Figure 3A. The majority of interactions between fibrinogen-coated beads and PIP5KI $\gamma^{-1-}$ megakaryocytes resulted in tether formation, yet this phenomenon was rarely seen in wild-type megakaryocytes or in PIP5KI $\gamma^{+/-}$megakaryocytes. There was a strong correlation between cells that had membrane blebs and those that allowed for the formation of membrane tethers. In contrast to the loss of the PIP5KI $\gamma$ isoform, loss of PIP5KI $\beta$ did not result in membrane tethers (Figure 3A and Supplemental Movie 6).

Primary sequence analysis suggested that PIP5KI $\alpha$ is more homologous with PIP5KI $\beta$ than it is with PIP5KI $\gamma$. Since PIP5KI $\beta$ is not required for the integrity of the connection between the cell membrane and the cytoskeleton, we hypothesized that this process would also not require PIP5KI $\alpha$. Although platelets and megakaryocytes contain very little PIP5KI $\alpha$, we analyzed the anchoring of the cell membrane to the cytoskeleton in megakaryocytes derived from the femurs of adult PIP5 $\mathrm{KI}^{-/-}$mice. As shown by the optical trap results displayed in Figure 3B, PIP5KI $\alpha$-null megakaryocytes did not have a defect in their ability to anchor their cell membrane to the underlying cytoskeleton. Together with the results

\section{Figure 2}

Loss of PIP5Kl $\gamma$ disrupts the association of the membrane to the cytoskeleton. Progenitor cells that were differentiated into megakaryocytes were analyzed for their ability to form membrane tethers by an optical trap pulling on a fibrinogen-coated membrane-attached bead. (A) Data traces of successive force signals produced during repeated contacts of a fibrinogen-coated latex bead with a wild-type megakaryocyte. PIP5Kl $\gamma^{+/+}$megakaryocytes possessed a cell membrane firmly bound to the cytoskeleton. At the moment of contact of a bead with a wild-type cell, the bead exerted a small positive compressive force on the cell (left part of upper panel). When the bead attached to the cell was pulled away, the force on the bead increased in the negative direction until the attachment bond was ruptured. At this point, the force rapidly returned to zero. If no attachment occurred, there was no negative rupture force signal. The lower panel shows a tracing derived from a PIP5KI $\gamma^{-/-}$ megakaryocyte. Initially, the bead freely oscillated and exerted no force signals while it approached the cell with 10-nm steps. After the fibrinogen-coated bead bound to the cell, the optical trap pulled it away. The first downward signal reflects the force needed to tear the membrane part off of the underlying cytoskeleton. Further positive and negative signals reflect cooscillation of the bead and the tethered membrane, which was typical for the PIPK $\gamma$-null megakaryocytes. (B) DIC microscopy demonstrated that PIP5KI $\gamma^{-1-}$ megakaryocytes formed membrane tethers by pulling on a murine fibrinogen-coated bead that was touched to the surface of the cell and then moved apart.

observed with PIP5KI $\beta$-null megakaryocytes, these data show that the PIP5KI $\gamma$ isoform fulfills a distinct role within these cells by contributing to the stable association between the cell membrane and the cytoskeleton.

PIP2 synthesized by PIP5KI $\gamma$, but not by PIP5KI $\beta$, is required for the anchoring of the cell membrane. We next analyzed whether $\mathrm{PIP}_{2}$ production within the PIP5 $\mathrm{KI}^{-/-}$megakaryocytes contributed to the blebbing phenotype and membrane flexibility observed in Figure 3A. Using retroviruses to express proteins within the cells, we found that the membrane phenotype was completely reverted by adding back GFP fused to wild-type PIP5KI $\gamma$ (Figure 3C). In contrast, the addition to these cells of a GFP fused to a catalytically inactive PIP5KI $\gamma$ mutant failed to revert the membrane-cytoskeleton defect. These data show that the lipid kinase activity of PIP5KI $\gamma$ regulates the membrane phenotype.

Although basal levels of $\mathrm{PIP}_{2}$ were normal in PIP5KI $\gamma^{-/}$megakaryocytes, we found that these cells did have mildly decreased PIP $_{2}$ levels following agonist stimulation (Figure 4). Surprisingly, PIP5KIBnull megakaryocytes that lack the membrane abnormality had at least as great of a defect in $\mathrm{PIP}_{2}$ synthesis (Figure 4). Furthermore, overexpression of PIP5KI $\beta$ in PIP5 $\mathrm{KI}^{-1-}$ megakaryocytes failed to rescue the cytoskeletal defect (Figure 3C). This demonstrates that production of $\mathrm{PIP}_{2}$ by PIP5KI $\gamma$ is required to preserve the integrity of the membrane cytoskeleton, but PIP $_{2}$ synthesized by PIP5KI $\beta$ in megakaryocytes does not contribute to this process. This suggests that local PIP 2 synthesis by PIP5KI $\gamma$ is required to preserve the structural integrity of the cellular membrane within these cells.

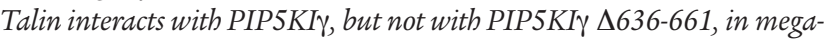
karyocytes. Observations initially made by Ling et al. and Di Paolo et al. demonstrated that PIP5KI $\gamma$ can associate with talin, a protein involved in cell membrane attachment to the environment $(17,18)$. The critical region of PIP5KI $\gamma$ essential for the association with talin has been localized to the terminal 26 amino acids of PIP5KI $\gamma$ and was absent in a naturally occurring splice variant of this enzyme. This splice variant, PIP5KI $\gamma \Delta 636-661$ has kinase 

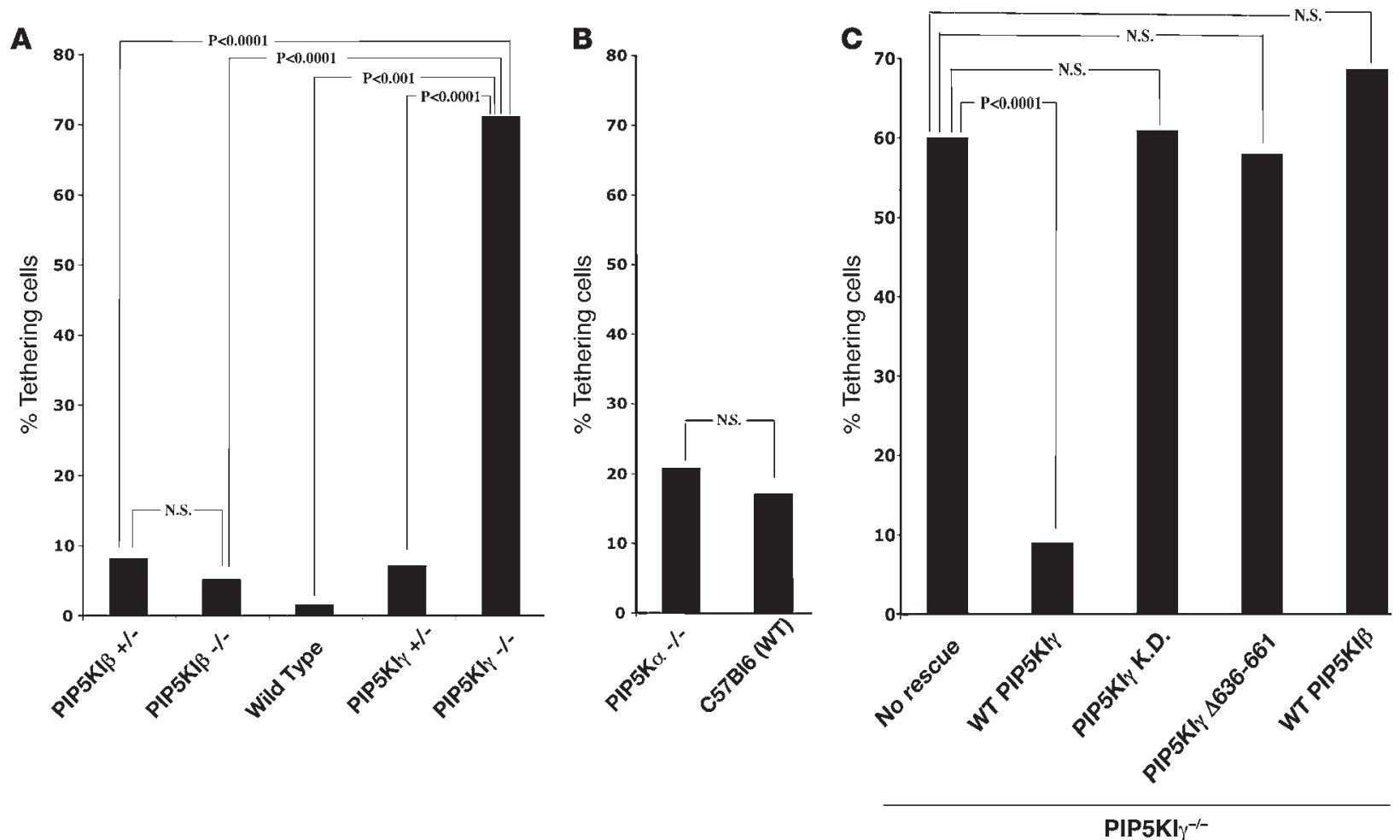

Figure 3

PIP5Kl $\gamma$, but not other PIP5KI isoforms, is required for stable interaction between the membrane and the cytoskeleton. (A) The ability of different genotypes of megakaryocytes to form membrane tethers is shown. The total number of interactions analyzed for each cell genotype were: PIP5KI $\gamma^{-1-}$, 91; PIP5KI $\gamma^{+-}$, 68; wild-type, 11; PIP5KI $\beta^{+--}, 12$; and PIP5KI $\beta^{-1-}$, 22. (B) The ability of megakaryocytes derived from adult PIP5KI $\alpha$-null or control mice to form membrane tethers is shown. For these experiments, the number of interactions analyzed were 345 (PIP5Kl $\alpha^{-1-}$ ) and 83 (wild-type). (C) Shown is the tether formation in a PIP5KI $\gamma^{-1-}$ megakaryocyte rescued by infection with retroviruses that induced expression of GFP fused to either wild-type PIP5Kl $\gamma(n=151)$, a catalytically inactive mutant PIP5Kl $\gamma$ D316A R447A K448A (PIP5Kl $\gamma$ KD, $n=126)$, the PIP5Kl $\gamma$ splice variant (PIP5Kl $\gamma \Delta 636-661, n=132$ ) lacking the terminal 26 -amino acid talin binding domain, or PIP5KI $\beta(n=71)$. The control bar on the left (no rescue) shows the tether formation in PIP5KI $\gamma^{-1-}$ megakaryocytes infected with empty retrovirus $(n=15)$. The statistical analysis was performed using the $\chi^{2}$ test.

activity comparable to the full-length protein (17). GFP fused to either full-length wild-type PIP5KI $\gamma$ or PIP5KI $\gamma$ splice variant $\Delta 636-661$ demonstrated grossly similar membrane-associated distributions when expressed in primary megakaryocytes (data not shown). We analyzed whether the talin-binding region of PIP5KI $\gamma$ was critical for its ability to promote a stable association of the cell membrane with the underlying cytoskeleton. In cells expressing PIP5KI $\gamma \Delta 636-661$, membrane tethers could be formed with fibrinogen-coated beads just as readily as in PIP5KI $\gamma^{-/-}$cells (Figure $3 C)$. Therefore, expression of the PIP5KI $\gamma$ splice variant $\Delta 636-661$ failed to rescue the membrane-cytoskeleton defect in PIP5KI $\gamma^{-1-}$ cells. This indicates that the PIP5KI $\gamma$ variant that cannot bind talin also does not contribute to the stable association between the cell membrane and the cytoskeleton.

Loss of talin 1 mimics the PIP5KI $\gamma^{-/-}$phenotype. The data show that both the catalytic activity of PIP5KI $\gamma$ and its ability to colocalize with talin are critical to maintain the integrity of the connection between the cell membrane and the cytoskeleton. This suggests a model by which PIP5KI $\gamma$ synthesizes PIP $_{2}$ in proximity to talin, and the local synthesis of $\mathrm{PIP}_{2}$ then regulates the ability of talin to anchor the cell membrane to the cytoskeleton. If this model is correct, then it stands to reason that loss of talin should create a membrane defect similar to that induced by the loss of PIP5KI $\gamma$.
Talin 1 is the only talin isoform in platelets and megakaryocytes (31). Mice homozygous for a conditional loss of the talin 1 gene $\left(T \ln 1^{f l f l}\right)$ have been previously described (32). These animals were crossed with transgenic mice containing Cre recombinase under the control of the megakaryocyte- and platelet-specific PF4 promoter to generate mice lacking talin 1 only in these cells $(33,34)$. We expanded megakaryocytes harvested from femurs of $T \ln 1^{f l / f l}$; PF4Cre ${ }^{+}$and Tln $1^{f l f f l}$ PF4Cre $e^{-}$mice. For comparison, megakaryocytes derived from femurs of the parental C57BL/6 line were also expanded. As predicted, $T \ln 1^{f l / f l} ; \mathrm{PF} 4 \mathrm{Cr} e^{+}$megakaryocytes had no talin 1 detected on immunoblots (Figure $5 \mathrm{~A}$ ). Interestingly, $T \ln 1^{f / f l}$; PF4Cre ${ }^{-}$megakaryocytes had significantly reduced talin 1 when compared with C57BL/6 wild-type megakaryocytes (Figure 5A) but still more talin 1 than was present in $T \ln 1^{f l / f l} ; P F 4 C r e^{+}$cells (Figure $5 \mathrm{~A})$. Although the cause of the hypomorphic phenotype in $T \ln 1^{f / f l}$; PF4Cre ${ }^{-}$megakaryocytes is unclear, it is potentially attributable to impaired transcriptional efficiency induced by the loxP site in the 5 ' untranslated region of the Tln 1 gene. This hypomorphic phenotype of the $T \ln 1^{f l / f l} ; P F 4 C r e^{-}$megakaryocytes allowed a comparison of cells expressing normal, low, or absent levels of talin 1 expression.

$T \ln 1^{f l / f l} ; \mathrm{PF} 4 \mathrm{Cr} \mathrm{C}^{+}$megakaryocytes that completely lacked talin 1 were morphologically similar to PIP5 $\mathrm{KI}{ }^{-/-}$cells and displayed a blebbing phenotype (Figure 5B). Experiments using the optical 

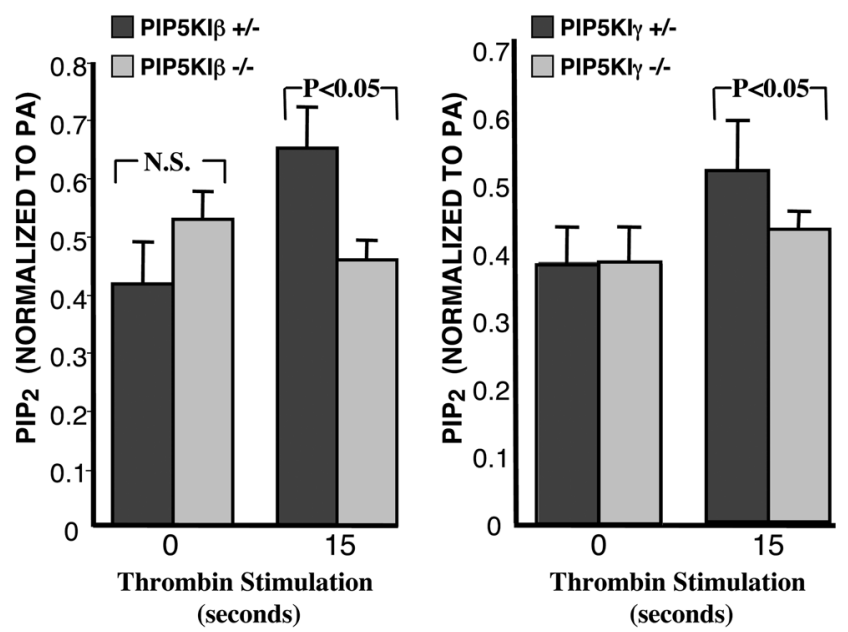

trap confirmed that talin1-null cells have impaired anchoring of the cell membrane to the underlying cytoskeleton (Figure 5C). Remarkably, $\operatorname{Tln} 1^{f l / f l} ; P F 4 C r e^{-}$megakaryocytes that had low levels of talin 1 expression had an intermediate phenotype in the optical trap and sometimes allowed for the formation of membrane tethers (Figure 5C). These results are consistent with the model that talin is part of the mechanism by which PIP5KI $\gamma$ regulates the association of the cell membrane with the cytoskeleton.

\section{Discussion}

These data demonstrate that PIP5KI isoforms have overlapping, but not completely redundant, functions within cells. Loss of either PIP5KI $\beta$ or PIP5KI $\gamma$ led to a global decrease in PIP $_{2}$ synthesis within megakaryocytes. However, only PIP5KI $\gamma$ was required for the attachment of the plasma membrane to the underlying cytoskeleton. The interaction between the cell membrane and the cytoskeleton was critical for the extension and contraction of membrane extensions such as filopodia and lamellipodia.

This observation suggests 2 alternative hypotheses as to why PIP5KI $\beta$ does not completely compensate for the loss of PIP5KI $\gamma$. First, activation of specific PIP5KI isoforms may be regulated differently. Local signals generated around regions of rupture of the plasma membrane from the cytoskeleton might stimulate PIP5KI $\gamma$ but not the other PIP5KI isoforms. Regional changes in hydrostatic pressure could serve to generate such a signal (35). In this model, PIP5KI $\gamma$ replaces the $\mathrm{PIP}_{2}$ that has been locally utilized by its interactions with $\mathrm{PIP}_{2}$-binding proteins or $\mathrm{PIP}_{2}$-modifying enzymes. In addition to PIP5KI $\gamma$ rapidly synthesizing PIP $_{2}$ at a rate that balances the local consumption by enzymes (phospholipases, phospholipid kinases, and inositol phosphatases), this model assumes that PIP5KI $\gamma$ can also synthesize enough PIP $_{2}$ to compensate for the regional loss of the phospholipid due to diffusion within the membrane.

An alternative hypothesis to explain our data is that different PIP5KI isoforms contribute to compartmentalized pools of $\mathrm{PIP}_{2}$ that control different aspects of platelet and megakaryocyte actin dynamics. It is possible that PIP5KI $\alpha$ and PIP5KI $\beta$ synthesize the pool of $\mathrm{PIP}_{2}$ that contributes to general second messenger formation, while PIP5KI $\gamma$ generates $\mathrm{PIP}_{2}$, which is required for the stable association of the membrane with the cytoskeleton.

Hinchliffe et al. were among the first to suggest that independent pools of $\mathrm{PIP}_{2}$ may contribute to distinctly different cellular func-

\section{Figure 4}

Loss of either PIP5KI $\beta$ or PIP5Kl $\gamma$ impairs $\mathrm{PIP}_{2}$ synthesis. PIP5KI $\beta^{-/-}$ and PIP5KI $\gamma^{-1-}$ progenitor cells were differentiated into megakaryocytes and incubated for 2 hours with free ${ }^{32} \mathrm{P}$ prior to stimulation with thrombin. Cells were lysed, fractionated by thin-layer chromatography, and analyzed for their relative $\mathrm{PIP}_{2}$ concentrations. Data was normalized to the concentration of PA. The graph shows the mean \pm SEM for 6 experiments.

tions (36). $\mathrm{PIP}_{2}$ concentration at the plasma membrane is relatively constant, although regional increases in $\mathrm{PIP}_{2}$ have been speculated to occur in actin-rich membrane ruffles and sites of phagocytosis $(22,23)$. Local synthesis of 3-phosphorylated phosphoinositides generated by phosphatidylinositol-3'-kinase exert biologic effects regionally by establishing gradients of specific phospholipids (37). Consistent with this model of regional phosphoinositide synthesis, it is conceivable that gradients of $\mathrm{PIP}_{2}$ could also contribute to actin dynamics at the leading edge of cells.

Some investigators have proposed that local synthesis of $\mathrm{PIP}_{2}$ cannot keep up with diffusion. The argument that $\mathrm{PIP}_{2}$ will diffuse away faster than it can be produced presumes that all $\mathrm{PIP}_{2}$ is unbound and free to diffuse. However, numerous proteins situated proximal to the cytoskeleton, including many actin-binding proteins, can bind and retain $\mathrm{PIP}_{2}$. Some of these proteins, such as GAP43, MARCKS, and Cap23, could trap PIP 2 in the regions of local synthesis $(38,39)$. Recently, Cho et al. measured the lateral diffusion coefficient for fluorescently labeled PIP and $\mathrm{PIP}_{2}$ delivered by patch pipettes to small areas of membrane within cells (40). Using this model, the diffusion coefficient of $\mathrm{PIP}_{2}$ was determined to be $0.00039 \mu \mathrm{m}^{2} / \mathrm{s}$, a coefficient over 10,000 -fold lower than that of PIP. This demonstrates that within the cellular environment, the lateral diffusion of $\mathrm{PIP}_{2}$ can be quite slow. Thus local synthesis of this phospholipid could have significant regional effects. Given the discrete localization of PIP5KI $\gamma$ to talin-rich structures $(17,18$, 41-44) and the disparate contribution of PIP5KI isoforms to PIP $_{2}$ dependent cytoskeletal integrity (Figure 3 and Supplemental Figures 5 and 6), our data support the model that local PIP 2 synthesis by PIP5KI $\gamma$ regulates regional cytoskeletal dynamics. At this point, we can not state whether our findings are explained by PIP5KI $\gamma$ synthesizing a true compartmentalized pool of $\mathrm{PIP}_{2}$, or whether PIP5KI $\gamma$ can locally synthesis $\mathrm{PIP}_{2}$ at a rate that is able to compensate for regional consumption and membrane diffusion.

Our observations complement data first proposed by Sheetz and colleagues, which demonstrated that marked disruption of $\mathrm{PIP}_{2}$ by overexpression of phospholipid-sequestering polypeptides or inositol phosphatases, or by pharmacologic activation of phospholipase $\mathrm{C}$, reduces the adhesion energy between the cell membrane and the cytoskeleton $(29,45)$. The structural mediators of this $\mathrm{PIP}_{2}$-driven signal are less clear, but candidate proteins are vinculin, talin, and filamin. $\mathrm{PIP}_{2}$ can directly associate with the tail region of vinculin and thereby activate vinculin to promote the connection between integrins and the actin cytoskeleton (46). Because of its proximity to focal adhesions, PIP5KI $\gamma$ could be poised to locally regulate this function of vinculin.

It is also possible that PIP5K $\gamma$ is required to regulate the linkage between filamin and the cytoskeleton. Filamin is a widely expressed protein that can promote high-angle branching of actin filaments and contributes to the regulation of integrins $(28,47$, 48). Stossel and colleagues have demonstrated that filamin contributes to the linkage between actin and the cell membrane (49). 
A

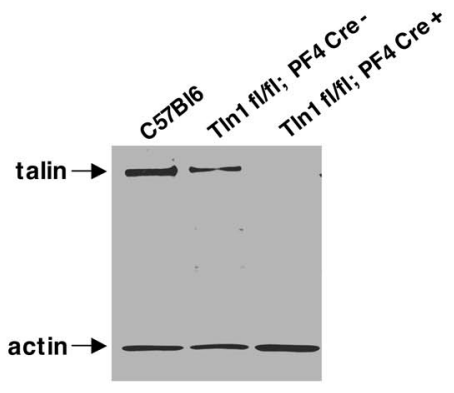

B

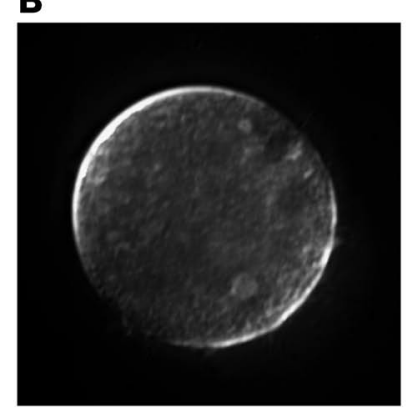

C57BI6

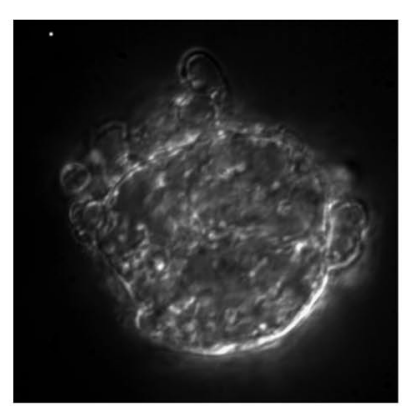

Tln1 flffl; PF4 Cre +

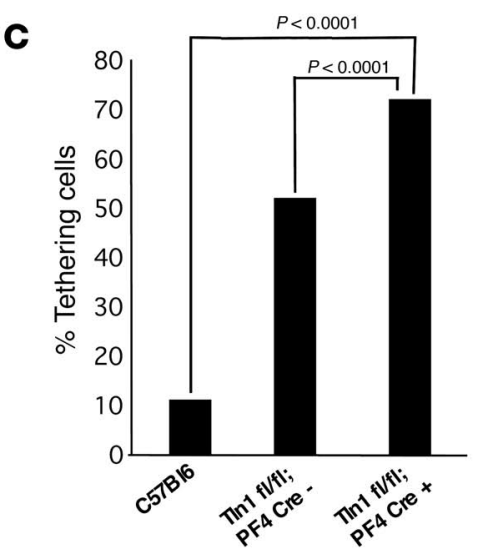

Furthermore, absence of filamin A in a melanoma cell line leads to a blebbing phenotype similar to that seen in PIP5K $\gamma$-null cells. Filamin A can also compete with talin for binding to some cell surface receptors (50). Since PIP5K $\gamma$ can directly bind to talin, it may be well positioned to locally synthesize $\mathrm{PIP}_{2}$, which might regulate filamin-talin dynamics.

Talin is another candidate effector proposed for PIP5KI $\gamma$. Talin has been demonstrated to bind $\mathrm{PIP}_{2}$, integrins, and actin (51). Talin, like other FERM family members, binds $\mathrm{PIP}_{2}$ and helps to link the actin cytoskeleton with the cell membrane (52-54). Previous reports have shown that, under certain circumstances, PIP5KI $\gamma$ can associate with talin $(17,18,41-43)$. The structures of several talin fragments interacting with peptides corresponding to PIP5KI $\gamma$ have recently been published $(44,55)$. Consistent with this interaction between full-length PIP5KI $\gamma$ and talin, we have found that expression of the shorter splice variant of PIP5KI $\gamma$ that fails to associate with talin in megakaryocytes is also insufficient to rescue the phenotype observed in PIP5 $\mathrm{KI}^{-/-}$cells (Figure 3). Furthermore, it is remarkable that talin1-null megakaryocytes display a membrane defect identical to cells lacking PIP5KI $\gamma$. This

\section{Figure 5}

Talin1-null megakaryocytes mimic cells lacking PIP5KI $\gamma$. Megakaryocytes were derived from the femurs of adult wild-type mice (C57BL/6) or from conditionally targeted talin 1 mice with ( $\left.T / n 1^{f|l| f \mid} ; P F 4 C r C^{+}\right)$or with-

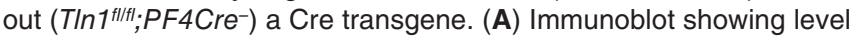
of talin and actin expression in megakaryocytes of different genotypes. (B) DIC images of wild-type (C57BL/6) and talin1-null (TIn $1^{\text {fllftl }}$ PF4Cre ${ }^{+}$) megakaryocytes demonstrate that loss of talin1 results in the formation of membrane blebs. (C) The ability of different megakaryocytes to form membrane tethers is shown. For these experiments, the number of cells analyzed was C57BL/6 $(n=66), \operatorname{TIn}^{\mathrm{fllffl} ;}$ PF $4 \mathrm{Cre}-(n=227)$, and TIn $1^{\text {fl/fli }}: P F 4 \mathrm{Cre}^{+}(n=178)$. The results show that the stable anchoring of the cell membrane to the cytoskeleton correlates with talin levels.

finding is consistent with work published by M. Sheetz and colleagues analyzing talin1-null fibroblasts $(54,56)$. Thus in contrast to other PIP5KI isoforms, PIP5KI $\gamma$ may be uniquely poised to generate locally high concentrations of $\mathrm{PIP}_{2}$ that regulate talin. Our data support this model that talin is vital for the ability of PIP5KI $\gamma$ to connect the cytoskeleton with the cell membrane.

Finally, could the ability to form membrane tethers contribute to the normal biology of cells? Recently, Ruggeri and colleagues demonstrated that under conditions of physiologic shear, platelets adherent to immobilized von Willebrand Factor stretched and formed tethers of membrane (57). This process ultimately results in the rupture of the tethers to release microparticles of membrane containing exposed phosphatidylserine. The exposure of platelet membrane phosphatidylserine is a critical aspect of the coagulation cascade. It is possible that regulation of microparticle formation is indirectly mediated in part by PIP2 synthesized by PIP5KI $\gamma$.

Work done by the S. Jackson group has demonstrated that platelets form membrane tethers as they are allowed to adhere to a matrix composed of von Willebrand Factor (58-60). Although these tethers are only present transiently, they are speculated to slow the sliding of platelets along this matrix enough to allow receptors to bind and support firm adhesion. Therefore, platelet tether formation could be a critical step in both platelet-matrix and platelet-platelet adhesive interactions. Whether PIP5KI $\gamma$ or $\mathrm{PIP}_{2}$ directly contribute to this phenomenon is not yet known. It is conceivable that downregulation of PIP5KI $\gamma$-mediated PIP2 synthesis allows tether formation and thereby supports platelet adhesion.

In summary, our data demonstrate that PIP5KI $\gamma$-mediated PIP 2 production plays a central role in cytoskeletal dynamics. The PIP5KI $\gamma^{-/-}$phenotype could be attributable to either deficient binding of $\mathrm{PIP}_{2}$ to cytoskeletal regulating proteins or insufficient phospholipid second messenger formation. Future studies of these phospholipid signals should provide greater insights and understanding of fundamental cytoskeletal dynamics.

\section{Methods}

Targeted PIP5KI $\alpha$, PIP5KI $\beta$, PIP5KI $\gamma$, and talin 1 murine lines. Targeting of the PIP5KI $\alpha$ and PIP5KI $\gamma$ genes and conditional targeting of the talin1 $\left(T \ln 1^{f / f l}\right)$ genes have been previously described $(26,32,61)$. To generate megakaryocyte and platelet-specific talin1-null animals, $T \ln 1^{f l / f l}$ mice were paired with mice transgenic for Cre recombinase under the control of PF4 (33). Berkeley Bay Genomics Group provided ES cell lines (XE248) containing disruption of one allele of the PIP5KI $\beta$ gene by $\beta$-geo random insertion mutagenesis (62). Using PCR-, Southern blotting-, and sequencing-based strategies, we identified the specific site of insertion of the $\beta$-geo cassette within the first intron of this gene. Generation of chimeric mice was per- 
formed at the Transgenic Core Facility at the University of Pennsylvania. PIP5KI $\beta$-null mice were born and appeared developmentally normal. A complete description of these mice will be described elsewhere. RT-PCR and immunoblotting indicate that the PIP5KI $\beta$-gene trap induces a complete null mutation (data not shown).

Culture of megakaryocytes. The yolk sacs of E10.5 embryos were dissected and disaggregated with collagenase. Hematopoietic progenitor cells were differentiated with thrombopoietin over 5 days into promegakaryocytes and megakaryocytes. In experiments using PIP5KI $\alpha$ - or talin-null cells, megakaryocytes were harvested from the femurs of adult mice. In an alternative method, G418-selected PIP5KI $\beta^{-/-}$or PIP5 $\mathrm{KI} \gamma^{-/-}$ES cells were differentiated into megakaryocytes as described by Shattil and colleagues (63). For some experiments, megakaryocytes were infected with an adenovirus or retrovirus that directed the expression of GFP-fusion proteins. Antitalin $8 \mathrm{~d} 4$ and anti-actin AC5 antibodies used for immunoblotting of megakaryocyte lysates were from Sigma-Aldrich.

Confocal microscopy. Megakaryocytes were layered onto opaque fibrinogen-coated DT4 dishes. Cells were imaged at $37^{\circ} \mathrm{C}$ under a $\times 60 / 1.4 \mathrm{NA}$ oil immersion lens (Nikon) using an Ultraview-LCI spinning disk confocal (PerkinElmer) attached to a Nikon TE-300 inverted light microscope. The Vybrant DiO (Invitrogen) or GFP was excited with the 488-nm line of the Argon ion laser (Melles-Griot), the RGB Dichroic mirror was used to transmit the laser light and reflect the emitted light, and a 525/50-nm barrier filter was used to reduce background fluorescence. Images were acquired using the temporal mode of the Imaging Suite software version 4.0 (PerkinElmer) with an Orca-ER digital CCD camera (Hammamatsu Photonics). Images were typically collected at 20-second intervals.

Membrane tethering using laser tweezers. A custom-built laser tweezers setup was used to form membrane tethers by pulling on a fibrinogen-coated bead that was touched repeatedly to the surface of a cell (64). Mouse fibrinogen (Sigma-Aldrich) was covalently bound to $1.87-\mu \mathrm{m}$ carboxylate-modified latex beads (Bangs Laboratories). The mixture of the cells and the beads was inserted into a flow microscope chamber in the culture medium containing $1 \mathrm{mM} \mathrm{MnCl}_{2}$ to activate the integrins, and the cells were allowed to settle on the polylysine-coated bottom of the chamber for 5-10 minutes. Near a cell that was firmly attached to the bottom, a bead was trapped and the microscope focus was adjusted so that the bead and cell centers were approximately the same distance from the bottom surface. The position of the laser trap was then oscillated in a triangular waveform with a frequency of $1 \mathrm{~Hz}$ and a constant peak-to-peak amplitude of $2 \mu \mathrm{m}(0.2 \mathrm{pN} / \mathrm{nm}$ trap stiffness; loading rate, $800 \mathrm{pN} / \mathrm{s}$ ). The bead position was sensed and digitized at the rate of 2,000 scans per second. Control and calibration of the instrument and data analysis were carried as described previously (64). The frequency of tether formation was calculated by pooling together data from all experiments performed with cells having the same genotype.

In the rescue experiments, fusion proteins composed of GFP and various PIP5KI polypeptides were added back to PIP5 $\mathrm{KI}^{-/-}$megakaryocytes by use of retroviruses. Inspection of GFP fluorescence indicated heterogeneity of expression levels within each batch of analyzed cells. Because of this heterogeneity, a reviewer blinded to the genotypes analyzed only GFP-bright cells in the optical trap-based model system.

Statistics. The $\chi^{2}$ test was used to compare the incidence of tether formation across the groups of cells of different genetic types. $P$ values of less than 0.05 were considered statistically significant.

\section{Acknowledgments}

We gratefully acknowledge the technical help of Edward Williams of the Diabetes Research Center and Jean Richea of the Transgenic Core (all at the University of Pennsylvania). We also thank Mitchell Weiss and David Stachura for the recombinant thrombopoietin and Warren Pear and the retrovirology core for their services and reagents. These studies were supported in part by funds from the $\mathrm{NIH}$ (to C.S. Abrams) and the Wellcome Trust (to D.R. Critchley).

Received for publication October 16, 2007, and accepted in revised form November 26, 2007.

Address correspondence to: Charles S. Abrams, HematologyOncology Division, University of Pennsylvania School of Medicine, 421 Curie Blvd., Biomedical Research Building II/III, \#912, Philadelphia, Pennsylvania 19104, USA. Phone: (215) 898-1058; Fax: (215) 573-7400; E-mail: abrams@mail.med.upenn.edu.
1. Hokin, M.R., and Hokin, L.E. 1953. Enzyme secretion and the incorporation of P32 into phospholipides of pancreas slices. J. Biol. Chem. 203:967-977.

2. Toker, A. 1998. The synthesis and cellular roles of phosphatidylinositol 4,5-bisphosphate. Curr. Opin. Cell Biol. 10:254-261.

3. Doughman, R.L., Firestone, A.J., and Anderson, R.A. 2003. Phosphatidylinositol phosphate kinases put PI4,5P(2) in its place. J. Membr. Biol. 194:77-89.

4. Oude Weernink, P.A., Schmidt, M., and Jakobs, K.H. 2004. Regulation and cellular roles of phosphoinositide 5-kinases. Eur. J. Pharmacol. 500:87-99.

5. Malm, B., Larsson, H., and Lindberg, U. 1983. The profilin--actin complex: further characterization of profilin and studies on the stability of the complex. J. Muscle Res. Cell Motil. 4:569-588.

6. Anderson, R.A., and Marchesi, V.T. 1985. Regulation of the association of membrane skeletal protein 4.1 with glycophorin by a polyphosphoinositide. Nature. 318:295-298.

7. Lassing, I., and Lindberg, U. 1985. Specific interaction between phosphatidylinositol 4,5-bisphosphate and profilactin. Nature. 314:472-474.

8. Burn, P., Rotman, A., Meyer, R.K., and Burger, M.M. 1985. Diacylglycerol in large alpha-actinin/actin complexes and in the cytoskeleton of activated platelets. Nature. 314:469-472.

9. Janmey, P.A., and Stossel, T.P. 1987. Modulation of gelsolin function by phosphatidylinositol 4,5-bisphosphate. Nature. 325:362-364.
10. Yin, H.L., and Janmey, P.A. 2003. Phosphoinositide regulation of the actin cytoskeleton. Annu. Rev. Physiol. 65:761-789.

11. Ishihara, H., et al. 1996. Cloning of cDNAs encoding two isoforms of 68 -kDa type I phosphatidylinositol-4-phosphate 5-kinase. J. Biol. Chem. 271:23611-23614.

12. Loijens, J.C., and Anderson, R.A. 1996. Type I phosphatidylinositol-4-phosphate 5-kinases are distinct members of this novel lipid kinase family. J. Biol. Chem. 271:32937-32943.

13. Ishihara, H., et al. 1998. Type I phosphatidylinositol-4-phosphate 5-kinases. Cloning of the third isoform and deletion/substitution analysis of members of this novel lipid kinase family. J. Biol. Chem. 273:8741-8748.

14. Stephens, L.R., Hughes, K.T., and Irvine, R.F. 1991. Pathway of phosphatidylinositol $(3,4,5)$-trisphosphate synthesis in activated neutrophils. Nature. 351:33-39.

15. Doughman, R.L., Firestone, A.J., Wojtasiak, M.L., Bunce, M.W., and Anderson, R.A. 2003. Membrane ruffling requires coordination between type Ialpha phosphatidylinositol phosphate kinase and Rac signaling. J. Biol. Chem. 278:23036-23045.

16. Padron, D., Wang, Y.J., Yamamoto, M., Yin, H., and Roth, M.G. 2003. Phosphatidylinositol phosphate 5-kinase Ibeta recruits AP- 2 to the plasma membrane and regulates rates of constitutive endocytosis. J. Cell Biol. 162:693-701.
17. Ling, K., Doughman, R.L., Firestone, A.J., Bunce, M.W., and Anderson, R.A. 2002. Type I gamma phosphatidylinositol phosphate kinase targets and regulates focal adhesions. Nature. 420:89-93.

18. Di Paolo, G., et al. 2002. Recruitment and regulation of phosphatidylinositol phosphate kinase type 1 gamma by the FERM domain of talin. Nature. 420:85-89.

19. Wenk, M.R., et al. 2001. PIP kinase Igamma is the major $\mathrm{PI}(4,5) \mathrm{P}(2)$ synthesizing enzyme at the synapse. Neuron. 32:79-88.

20. Mao, Y.S., and Yin, H.L. 2007. Regulation of the actin cytoskeleton by phosphatidylinositol 4-phosphate 5 kinases. Pflugers Arch. 455:5-18.

21. McLaughlin, S., Wang, J., Gambhir, A., and Murray, D. 2002. PIP(2) and proteins: interactions, organization, and information flow. Annu. Rev. Biophys. Biomol. Struct. 31:151-175.

22. Chatah, N.E., and Abrams, C.S. 2001. G-proteincoupled receptor activation induces the membrane translocation and activation of phosphatidylinositol-4-phosphate 5-kinase ialpha by a rac- and rhodependent pathway. J. Biol. Chem. 276:34059-34065.

23. Botelho, R.J., et al. 2000. Localized biphasic changes in phosphatidylinositol-4,5-bisphosphate at sites of phagocytosis. J. Cell Biol. 151:1353-1368.

24. Tolias, K.F., et al. 2000. Type Ialpha phosphatidylinositol-4-phosphate 5-kinase mediates Racdependent actin assembly. Curr. Biol. 10:153-156.

25. Yang, S.A., Carpenter, C.L., and Abrams, C.S. 2004. 
Rho \& Rho-kinase mediate thrombin induced PIP5K trafficking in platelets. J. Biol. Chem. 279:42331-42336.

26. Wang, Y., Lian, L., Golden, J., Morrisey, E.M., and Abrams, C.S. 2007. PIP5K $\gamma$ is required for cardiovascular and neuronal development. Proc. Natl. Acad. Sci. U. S. A. 104:11748-11753.

27. Di Paolo, G., et al. 2004. Impaired PtdIns(4,5)P2 synthesis in nerve terminals produces defects in synaptic vesicle trafficking. Nature. 431:415-422.

28. Flanagan, L.A., et al. 2001. Filamin A, the Arp $2 / 3$ complex, and the morphology and function of cortical actin filaments in human melanoma cells. J. Cell Biol. 155:511-517.

29. Raucher, D., et al. 2000. Phosphatidylinositol 4,5-bisphosphate functions as a second messenger that regulates cytoskeleton-plasma membrane adhesion. Cell. 100:221-228.

30. Evans, E., Heinrich, V., Leung, A., and Kinoshita, K. 2005. Nano- to microscale dynamics of P-selectin detachment from leukocyte interfaces. I. Membrane separation from the cytoskeleton. Biophys. J. 88:2288-2298.

31. Senetar, M.A., Moncman, C.L., and McCann, R.O. 2007. Talin 2 is induced during striated muscle differentiation and is targeted to stable adhesion complexes in mature muscle. Cell. Motil. Cytoskeleton. 64:157-173.

32. Moes, M., et al. 2007. The integrin binding site 2 (IBS2) in the talin rod domain is essential for linking integrin beta subunits to the cytoskeleton. J. Biol. Chem. 282:17280-17288.

33. Tiedt, R., Schomber, T., Hao-Shen, H., and Skoda, R.C. 2007. Pf4-Cre transgenic mice allow the generation of lineage-restricted gene knockouts for studying megakaryocyte and platelet function in vivo. Blood. 109:1503-1506.

34. Chen, Z., et al. 2007. The May-Hegglin anomaly gene MYH9 is a negative regulator of platelet biogenesis modulated by the Rho-ROCK pathway. Blood. 110:171-179.

35. Charras, G.T., Yarrow, J.C., Horton, M.A., Mahadevan, L., and Mitchison, T.J. 2005. Non-equilibration of hydrostatic pressure in blebbing cells. Nature. 435:365-369.

36. Hinchliffe, K.A., Ciruela, A., and Irvine, R.F. 1998. PIPkins1, their substrates and their products: new functions for old enzymes. Biochim. Biophys. Acta. 1436:87-104.

37. Haugh, J.M., Codazzi, F., Teruel, M., and Meyer, T. 2000. Spatial sensing in fibroblasts mediated by $3^{\prime}$ phosphoinositides. J. Cell Biol. 151:1269-1280.

38. Laux, T., et al. 2000. GAP43, MARCKS, and CAP23 modulate $\mathrm{PI}(4,5) \mathrm{P}(2)$ at plasmalemmal rafts, and regulate cell cortex actin dynamics through a common mechanism. J. Cell Biol. 149:1455-1472.

39. Gambhir, A., et al. 2004. Electrostatic sequestration of PIP2 on phospholipid membranes by basic/aromatic regions of proteins. Biophys. J. 86:2188-2207.

40. Cho, H., et al. 2005. Low mobility of phosphatidylinositol 4,5-bisphosphate underlies receptor specificity of Gq-mediated ion channel regulation in atrial myocytes. Proc. Natl. Acad. Sci. U. S. A. 102:15241-15246.

41. Ling, K., et al. 2003. Tyrosine phosphorylation of type Igamma phosphatidylinositol phosphate kinase by Src regulates an integrin-talin switch. J. Cell Biol. 163:1339-1349.

42. Lee, S.Y., et al. 2005. Regulation of the interaction between PIPKI gamma and talin by proline-directed protein kinases. J. Cell Biol. 168:789-799.

43. Bairstow, S.F., Ling, K., and Anderson, R.A. 2005. Phosphatidylinositol phosphate kinase type Igamma directly associates with and regulates Shp-1 tyrosine phosphatase. J. Biol. Chem. 280:23884-23891.

44. de Pereda, J.M., et al. 2005. Structural basis for phosphatidylinositol phosphate kinase type Igamma binding to talin at focal adhesions. J. Biol. Chem. 280:8381-8386.

45. Raucher, D., and Sheetz, M.P. 2001. Phospholipase C activation by anesthetics decreases membrane-cytoskeleton adhesion. J. Cell Sci. 114:3759-3766.

46. Chandrasekar, I., et al. 2005. Vinculin acts as a sensor in lipid regulation of adhesion-site turnover. J. Cell Sci. 118:1461-1472.

47. Gorlin, J.B., et al. 1990. Human endothelial actinbinding protein (ABP-280, nonmuscle filamin): a molecular leaf spring. J. Cell Biol. 111:1089-1105.

48. Calderwood, D.A., et al. 2001. Increased filamin binding to beta-integrin cytoplasmic domains inhibits cell migration. Nat. Cell Biol. 3:1060-1068.

49. Cunningham, C.C., Stossel, T.P., and Kwiatkowski, D.J. 1991. Enhanced motility in NIH 3 T3 fibroblasts that overexpress gelsolin. Science. 251:1233-1236.

50. Kiema, T., et al. 2006. The molecular basis of filamin binding to integrins and competition with talin. Mol. Cell. 21:337-347.

51. Martel, V., et al. 2001. Conformation, localization, and integrin binding of talin depend on its interaction with phosphoinositides. J. Biol. Chem. 276:21217-21227.

52. Horwitz, A., Duggan, K., Buck, C., Beckerle, M.C., and Burridge, K. 1986. Interaction of plasma membrane fibronectin receptor with talin - a transmembrane linkage. Nature. 320:531-533.

53. Calderwood, D.A., et al. 1999. The talin head domain binds to integrin beta subunit cytoplasmic tails and regulates integrin activation. J. Biol. Chem. 274:28071-28074.

54. Jiang, G., Giannone, G., Critchley, D.R., Fukumoto, E., and Sheetz, M.P. 2003. Two-piconewton slip bond between fibronectin and the cytoskeleton depends on talin. Nature. 424:334-337.

55. Kong, X., Wang, X., Misra, S., and Qin, J. 2006. Structural Basis for the Phosphorylation-regulated Focal Adhesion Targeting of Type Igamma Phosphatidylinositol Phosphate Kinase (PIPKIgamma) by Talin. J. Mol. Biol. 359:47-54.

56. Giannone, G., Jiang, G., Sutton, D.H., Critchley, D.R., and Sheetz, M.P. 2003. Talin1 is critical for force-dependent reinforcement of initial integrincytoskeleton bonds but not tyrosine kinase activation. J. Cell Biol. 163:409-419.

57. Reininger, A.J., et al. 2006. Mechanism of platelet adhesion to von Willebrand factor and microparticle formation under high shear stress. Blood. 107:3537-3545.

58. Dopheide, S.M., Maxwell, M.J., and Jackson, S.P. 2002. Shear-dependent tether formation during platelet translocation on von Willebrand factor. Blood. 99:159-167.

59. Maxwell, M.J., Dopheide, S.M., Turner, S.J., and Jackson, S.P. 2006. Shear induces a unique series of morphological changes in translocating platelets: effects of morphology on translocation dynamics. Arterioscler. Thromb. Vasc. Biol. 26:663-669.

60. Maxwell, M.J., et al. 2007. Identification of a 2-stage platelet aggregation process mediating shear-dependent thrombus formation. Blood. 109:566-576.

61. Sasaki, J., et al. 2005. Regulation of anaphylactic responses by phosphatidylinositol phosphate kinase type I \{alpha\}. J. Exp. Med. 201:859-870.

62. Skarnes, W.C. 2000. Gene trapping methods for the identification and functional analysis of cell surface proteins in mice. Methods Enzymol. 328:592-615.

63. Eto, K., Leavitt, A.L., Nakano, T., and Shattil, S.J. 2003. Development and analysis of megakaryocytes from murine embryonic stem cells. Methods Enzymol. 365:142-158.

64. Litvinov, R.I., Bennett, J.S., Weisel, J.W., and Shuman, H. 2005. Multi-step fibrinogen binding to the integrin (alpha)IIb(beta) 3 detected using force spectroscopy. Biophys. J. 89:2824-2834. 